\title{
Estimating Average Flow Delay in AQM Router
}

\author{
Ming Liu, Wen-hua Dou, and Rui Xiao \\ Computer College, National University of Defense Technology \\ Hunan, 410073, P.R. China \\ liutomorrowahotmail.com
}

\begin{abstract}
Delay measurement plays an important role in network QOS control. Much work has been done about the measurement of end-to-end delay, this paper describes a mechanism estimating flow Number and average delay in AQM router in the Internet. This estimate is obtained without collecting or analyzing state information on individual flows.
\end{abstract}

\section{Introduction}

The estimation of connections and delay is useful for network QOS control and resource management. Much work has been done about the measurement of end-to-end delay and several models were proposed ${ }^{[1]}$. Variously, this paper describes a mechanism estimating flow Number and average delay in an $\mathrm{AQM}^{[2]}$ router in the Internet.

A linearized TCP/AQM dynamic model was developed ${ }^{[3]}$. The forward-path transfer function of the plant $P(s)=P_{\text {tcp }}(s) \times P_{\text {queue }}(s) \times e^{-s R_{0}}$ was given by

$$
P_{\text {tcp }}(s)=\left(\frac{R_{0} C^{2}}{2 N^{2}}\right) /\left(s+\frac{2 N}{R_{0}^{2} C}\right), \quad P_{\text {queиe }}(s)=\left(\frac{N}{R_{0}}\right) /\left(s+\frac{1}{R_{0}}\right)
$$

where $N$ is load factor (number of TCP connections), $R_{0}$ is round trip time, $C$ is the link capacity. The actuating signal produced by AQM controller also reflects the information of flows that traverse the router.

\section{Estimating Average Flow Delay in an AQM Router}

\subsection{Estimating the Flow Number}

By TCP/AQM model, we need estimate the flow number firstly. Several approaches have been proposed previously to estimate the number of active flows competing for bandwidth $^{[4,5,6]}$. Stabilized RED (SRED) compares the arrival packet with recently arrived packets in a 'Zombie List' and estimates the number of flows based on the hit probability. Hash Rate Estimation (HRE) uses a similar method while bases on the comparison of the incoming packet with the randomly selected backlogged packets in the queue. Flow Random Early Detection (FRED) estimates the number of flows that have packets queuing in the buffer. In FPIP, routers maintain a state table of long 
flows to record the arrival time (prevtime) of the packet arrived lately from each long flow.

The approaches described above are effective in specific environments, we use the reference of the method "hit and miss" here. The main idea is to compare, whenever a packet arrives at some buffer, the arriving packet with a randomly chosen packet that recently preceded it into the buffer.

Considering the following situation, there are $n$ flows passing through the router; $r_{i}$ and $p_{i}$ respectively denote the flow rate and the buffer occupancy of flow $i$. Intuitively, $r_{h i t}$ is the rate that the flow ID of incoming packet matches that of one randomly selected packet in the queue and $r_{\text {miss }}$ denotes the rate that two predescribed packet are not of the same flow. The active flow number $N_{a c t}$, are shown as:

$$
N_{\text {act }}=\frac{r_{\text {hit }}+r_{\text {miss }}}{r_{\text {hit }}}=\frac{1}{r_{\text {hit }}}, \quad r_{\text {hit }}=\sum_{i=1}^{n} r_{i} p_{i} / C ; \quad r_{\text {miss }}=\sum_{i=1}^{n} r_{i}\left(1-p_{i}\right) / C
$$

We calculate an estimate $r_{\text {hit }}$ for the hit frequency around the time of the arrival of packet. $($ Let $\operatorname{Hit}(\mathrm{t})=0$ if no hit and $\operatorname{Hit}(\mathrm{t})=1$ if hit):

$$
r_{\text {hit }}(k)=(1-\alpha) r_{h i t}(k-1)+\alpha H i t(k)
$$

with $0<\alpha<1$, here $\alpha=2.5 \times 10^{-4}$.

We use simple network topology with a single bottleneck link between r1 and $\mathrm{r} 2$ as depicted in Figure 1. C is $3750 \mathrm{pkt} / \mathrm{s}$ which corresponds to $15 \mathrm{Mbps}$ with an average packet size of 500B. Connections are established between $s_{i}$ and $d_{i}$. The propagation delay ranges uniformly between $40 \mathrm{~ms}$ and $220 \mathrm{~ms}$, our target queue length is 100 packets. 11 runs PI and supports ECN marking, while the other router runs Drop Tail, buffer size is 800 packets.

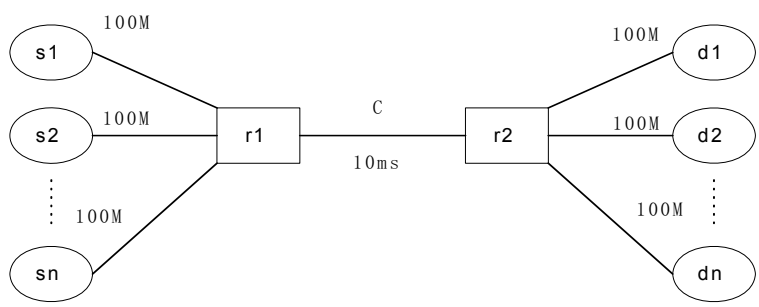

Fig. 1. Simple network topology

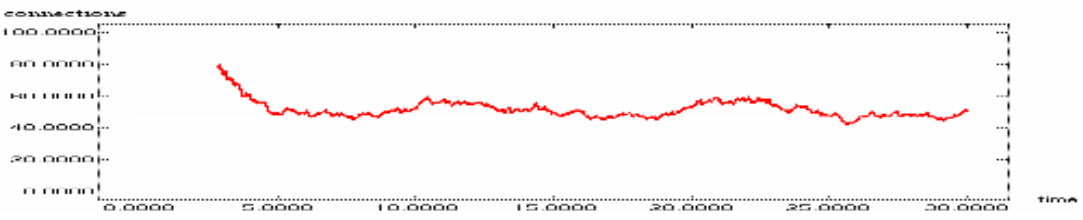

Fig. 2. the estimated flow number $\mathrm{N}_{\text {act }}$ in experiment 1 
In experiment 1 , the number of FTP flows is 50 at the beginning, the total simulation lasted for $30 \mathrm{~s}$. As shown in the figure, the estimated value $\mathrm{N}_{\text {act }}$ can converge to the real flow number.

In experiment 2, the number of FTP flows is 50 at the beginning, 50 FTP flows join the link 20 seconds later, 50 FTP flows join the link when t=40s, 50 FTP flows leave the link 30 seconds later, the total simulation lasted for 100s. As shown in the experiment result, the estimated value $\mathrm{N}_{\text {act }}$ can converge to the real flow number, and change with the real flow number $n$.

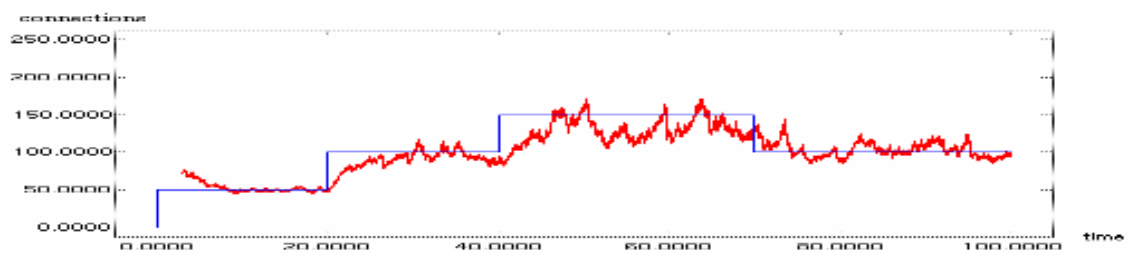

Fig. 3. the estimated flow number $\mathrm{N}_{\text {act }}$ in experiment 2

\subsection{Estimating the Average Flow Delay}

Using linearization techniques near equilibrium point of non-linear differential equation $^{[3]},\left(W_{0}, q_{0}, p_{0}\right)$ is the equilibrium point which meet

$$
\left.\frac{d W(t)}{d(t)}\right|_{\left(W_{0}, q_{0}, p_{0}\right)}=\frac{1}{R_{0}}-\frac{W_{0}^{2}}{2 R_{0}} p_{0}=0,\left.\quad \frac{d q(t)}{d(t)}\right|_{\left(W_{0}, q_{0}, p_{0}\right)}=\frac{N}{R_{0}} W_{0}-C=0
$$

$W_{0}, q_{0}, p_{0}$ can be calculated from: $W_{0}^{2} p_{0}=2, W_{0}=R_{0} C / N$, so

$$
R_{0}=q_{0} / C+T_{p}=\frac{\sqrt{2} N}{C \sqrt{p_{0}}}
$$

$p$ is probability of packet mark/drop decided by AQM modules, we use the exponentially weighted moving average mark/drop probability as our $p_{0}$ :

$$
p_{0}(k)=(1-\alpha) p_{0}(k-1)+\alpha p
$$

$0<\alpha<1$, here $\alpha=2.5 \times 10^{-5}$

We still use the network topology depicted in figure 1 . In experiment 3 , the propagation delay between the end and the router is $5 \mathrm{~ms}$, other settings are same as

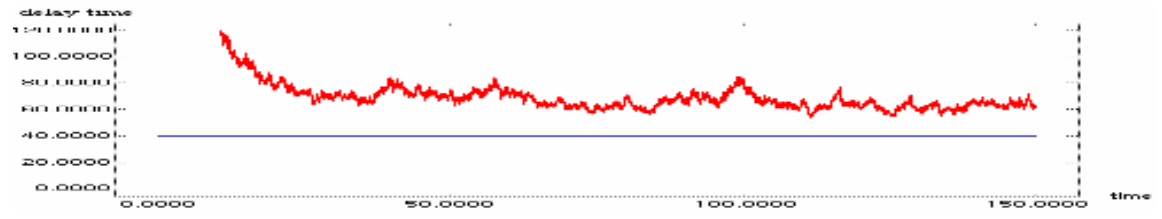

Fig. 4. The estimated delay time in experiment 3 
experiment 1 , so the propagation delay is $40 \mathrm{~ms}$, and the queueing delay is during $0 \sim 50 \mathrm{~ms}$. The number of FTP flows is 50 at the beginning, the total simulation lasted for $150 \mathrm{~s}$. As shown in the figure, the estimated delay time is within the range of real delay time.

In experiment 4 , the propagation delay between the end and the router is $10 \mathrm{~ms}$, other settings are same as experiment 3, so the propagation delay is $60 \mathrm{~ms}$, and the queueing delay is during $0 \sim 50 \mathrm{~ms}$. As shown in the figure, the estimated delay time is within the range of real delay time.

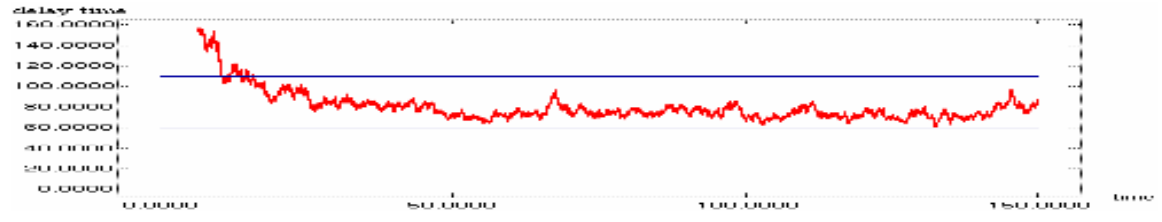

Fig. 5. The estimated delay time in experiment 4

\section{Conclusion}

This paper describes a mechanism estimating flow Number and average delay in a router in the Internet. This estimate is obtained without collecting or analyzing state information on individual flows.

There are also some limits with the estimating mechanism. It will take a period of time for the estimation to converge to the real value, this depends on the AQM scheme deployed in the router. Sometimes the estimated values are smaller than reality: if all flows have the same rate, the value $N_{\text {act }}$ equals the real value $n$, otherwise, the estimated number will be smaller; the estimated average delay will be smaller than reality when nonresponsive flows exist. Suitable adjustment of the estimation value need further attention.

\section{References}

1. G. Almes, S. Kalidindi, M. Zekauskas. A One-way Delay Metric for IPPM, RFC 2679, September 1999

2. B. Braden, D. Clark, J. Crowcroft, B. etc, Recommendations on Queue Management and Congestion Avoidance in the Internet, RFC2309, April 1998.

3. Vishal Misra, Wei-Bo Gong, and Don Towsley, Fluid-based Analysis of a Network of AQM Routers Supporting TCP Flows with an Application to RED[A], in Proceedings of ACM SIGCOMM 2000[C], Stockholm, Sweden, 2000.

4. Li J.S.and Leu M.S. Network Fair Bandwidth Share Using Hash Rate Estimation. Networks, 40(3):125-141. 2002.

5. Lin D and Morris R. Dynamics of Random Early Detection. In Proceedings of the ACM SIGCOMM 1997, pp.127-137. Cannes, France. 1997.

6. Ott T. J., Lakshman T.V. and Wong L.H. SRED: Stabilized RED.In Proceedings of the IEEE INFOCOM 1999, pp.1346-1355. New York, USA. 1999. 